\title{
Towards an Imperfect Robot for Long-term Companionship: Case Studies Using Cognitive Biases
}

\author{
Biswas, M. and Murray, J.C.
}

\begin{abstract}
The research presented in this paper aims to find out what affect cognitive biases play in a robot's interactive behaviour for the goal of developing human-robot long-term companionship. It is expected that by utilising cognitive biases in a robot's interactive behaviours, making the robot cognitively imperfect, will affect how people relate to the robot thereby changing the process of long-term companionship. Previous research carried out in this area based on human-like cognitive characteristics in robots to create and maintain long-term relationship between robots and humans have yet to focus on developing human-like cognitive biases and as such is new to this application in robotics. To start working with cognitive biases 'misattribution' and 'empathic gap' have been selected which have been shown to be very common biases in humans and as such play a role on human-human interactions and long-term relationships.
\end{abstract}

Index Terms - Human-Robot Interaction, Human-Robot Long-term Companionship, Cognitive Bias, Imperfect Robots, Misattribution, Empathic Gap.

\section{INTRODUCTION}

This paper seeks to influence robot-human interaction and communication with 'cognitive biases' to provide a more humanlike interaction process. Currently, most human-robot interaction is based on a set of well-ordered and structured rules, these repeat regardless of the person or social situation. This tends to provide an unrealistic interaction, which makes it difficult for humans to relate 'naturally' with the robot after a number of interactions. The 'problem', the robot appears to be 'too' perfect and as such, unnatural.

Apart from personality and characteristic traits, cognitive biases play an important role in basic human behavioral actions (Maccon, 1998). Robots on the other hand are still machines which are given a certain type of personality depending on its interaction with humans and behavioral characteristics (Meerbeek B, 2009). Hayoun (2014) raised an important question, "How can we interact with something 'more perfect' than we are?" As we know, making faults and misjudgements are common human characteristics, but robotic engineers and researchers are trying to create something that is more perfect than we are. Human-like cognitive imperfections have not been tested in robots and long-term human-robot relationships. Indeed it has not been investigated that if the robot makes human-like mistakes in their tasks then how does this affect the human-robot relationship? In this paper we introduce a model demonstrating cognitive biased behaviours in robots. It is hoped that this more natural system of interaction allows the human to build a stronger long-term social interaction with the robot.

The robots used in these experiments are ERWIN (Emotional Robot with Intelligent Networks) (Murray J, 2008) and MyKeepon (Kozima H, 2009). The robot's behavioral characteristics and biases were carefully chosen so that ERWIN could exhibit several prototypical facial expressions and, MyKeepon can express emotions to influence the interaction process. The robots were also described as cheerful and friendly by participants (Biswas M, 2014). The interaction process was developed to allow ERWIN to remember previous interactions with the specific participants to allow previously gained knowledge and conversations to be used in the hope of building a long-term relationship. Also, for MyKeepon we chose to reduce or increase its empathic behaviours to differentiate between two interactions so that the participants can recognize the behavioral changes and bias affects during interactions.

\section{PREVIOUS RESEARCH IN HRI}

Robots are being used in various fields including heavy industrial works, medical treatments, caregivers for elderly and Autistic children, rescue workers and in different research studies (Breazeal C, 2002). Robotics research has achieved a steady growth in recent years (Tobe F, 2014), and as a result of this, much research has been carried out in the field of human-robot interaction, as follows:

A social robot should be socially intelligent and should have sufficient social knowledge (Breazeal C, 2001). Dautenhahn (2007) investigated the identifying links between human personality and attributed robot personality where the team investigated human and robot personality traits as part of a human-robot interaction trial. Anthropomorphism is one of the popular approaches to create human like behaviours in others. Recent research by Bernt Meerbeek et al (2009) on iterative personality design process in robots which was based on Duffy's anthropomorphism idea (Duffy B, 2003). Reeves and Nass (1996) shown that users usually show biased driven certain personality traits to machines (PC \& others) and from that research they proposed 'user driven' mental model for the domestic robots. At Michigan State University research has been carried out on Extraversion, one of the most popular dimensions of the personality trait of the Big-five trait theory with a Sony AIBO focused on 'extrovert' and 'introvert' characteristics (Lee K, 2006). Their research found the same 
complimentary attraction effect between the participants and the robot dog.

All the above research focus on implementing human-like trait attributes in robots in order to develop human-robot companionship. But, outside the laboratory the real-time human-like interactions are still a challenge for the robots, yet social companion robots are unable to interact in continuous, extended social interactions beyond time-limits and still lack to adapt to users interactive behaviours based on previous interactions (Baxter P, 2012). In our past experiments (Biaswas M, 2014), we have noticed that participants were very interested and eager to meet with our robots for the first time, but their interests dropped after a few interactions. Such reasons could include the fulfilling of preliminary interests of the part of the participant's basic curiosity to the robot and getting a general idea of the robot's capabilities.

From the mentioned research, it can be said that, emotion expressions, anthropomorphic behaviours, personality traits in robots are not extending the relationship beyond time scale. To develop human-robot long-term companionship one might need to think about other human-like activities in robots to make the robot-human interactions as natural as humans' interactions. In our current research, we follow a simple and new idea to make the robot's interactive behaviours more familiar to humans, by developing cognitive biases in robots and as such making the robots as imperfect as humans.

\section{IMPERFECT ROBOT AND COGNITIVE BIAS}

Current social robots are able to anthropomorphize and mimic human actions but their actions are limited and may not provide sufficient reasons for people to create and maintain social relationship (Baxter P, 2012). But in human interactions, people usually meet with other humans and are able to form different kinds of relationships. From that, we raise a simple question, "What happens in human-human interaction which lacks in human-robot interaction that prevents a social relationship between the robot and human?'

In the research presented here, the term 'cognitively imperfect robot' refers to a robot which shows typical cognitive biased behaviours in its interactions with humans. Most of the current social robots show various human-like characteristics in their mode of interactions, but most of their humanlike cognitive characteristics are being used to make the robot more intelligent or smarter in its behaviour.

Sometimes a robot's social behaviours lacks that of a human's common characteristics such as, idiocracy, humour and common mistakes. Many robots are able to present social behaviours in human-robot interactions but unable to show human-like cognitively imperfect behaviours. Or 'human-like behaviours' are presented in such manner that participants cannot relate themselves with the robot. We understand that, a human's perception to the robot is closely related to the robot's behavioural actions used in communications; and for that, we expect that cognitive biases will make robot's behaviour human-like fallible which is known to human.

Studies suggest that various cognitive biases have a reasonable amount of influences on human thinking process to make misjudgements, mistakes and fallible activities (Baron, 2007). Such misjudgements, mistakes and fallible activities creates individual's social behaviour human-like cognitively imperfect (T. Michael, 1999). Bless et al (2004) suggested that cognitive biases can influence on human's behaviours towards positive or negative ways. Biases effect on individual's decision making, characteristics behaviours and social beliefs. In our understandings, such cognitive biases effect individual's general communicative behaviours in humanhuman interaction and that makes the interaction human-like natural. But, robots in the other hand, lack to present humanlike cognitive characteristics in human-robot interaction and that might prevent the interaction to become human-like natural. From the above understanding we have chosen two common cognitive biases, misattribution and empathy gap to work on our robots ERWIN and MyKeepon. We expect that cognitive imperfect behaviours presented by robots during the interactions could help humans to understand and relate with the robot more easily. Also it is expected, such interactions could help to create a bond between the human and robot effectively and as such would help to create a long-term human-robot companionship.

\section{THE EXPERIMENTS}

\section{A. Introduction}

Two sets of experiments have been completed with two robots and participants. The first set of experiments with ERWIN and a total of 30 participants, and the second set of experiments with Keepon and another 30 participants. The Misattribution bias was used in ERWIN's speech, and Empathy gap, gambler fallacy, conjunction biases were used in MyKeepon's behaviors to make it overly joys, over-sad and sometime unresponsive.

\section{B. ERWIN Experiments:}

ERWIN is a robot head with 6 degrees of freedom. It can move its head 360 degrees and also can tilt sideways. It has 2 cameras in for eyes and can express basic prototypical emotions such as, happy, sad, angry, surprise, shock or fear.

For ERWIN's part of the conversation, text-to-speech software 'Speakonia' was used which has small prosodic abilities while talking in long sentences. For the purpose of experiments, the Wizard of $\mathrm{Oz}$ methodology was adopted as the response of the robot here is not the important factor, but rather the human response is what is being measured.

The Wizard was monitoring the live reactions from the participant and was generating relevant emotions and conversational responses to ensure that the interaction flow stays adequate. Emotions were generated during the speech maintaining the conversational direction.

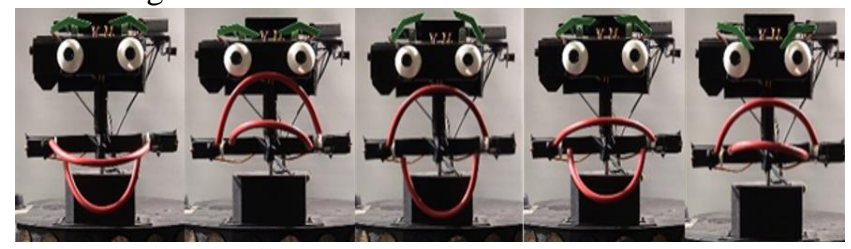

Fig. 1. Different emotions expressions by ERWIN. 


\begin{tabular}{|l|l|}
\hline $1^{\text {ts }}$ emotion expression & To greet the participant. \\
\hline $2^{\text {nd }}$ emotion expression & To continue the conversation \\
\hline
\end{tabular}

Fig 2.a. Emotions cues for ERWIN

ERWIN's expressions and speech cues were as follows:

\begin{tabular}{|l|l|l|}
\hline \multicolumn{1}{|c|}{$\rightarrow$ Start } & \multicolumn{1}{|c|}{ Action cue } & \\
\hline & Speech (1) & Greets \\
\hline & Expression (1) & Happy \\
\hline Wait for response (1) & & \\
\hline Receive the response (1) & & \\
\hline & Expression (2) & According to the response \\
\hline & Speech (2) & According to the response (1) \\
\hline & Expression (3) & According to the speech (2) \\
\hline Wait for response (2) & & \\
\hline
\end{tabular}

Fig 2.b. Expressions and Speech cues for ERWIN

There were three interactions with ERWIN for each participant. These three interactions were held in three different experiments and maintaining a time gap of several days to allow long-term affectivity in the participants.

The first experiment 'Introduction' was common for each participant to allow familiarization with the environment and robot. Step 1: Identification; the participants were asked to identify ERWINs different facial expressions from pictures to see if they could identify the expressions without meeting the robot. Step 2: Conversation; the robot started friendly conversation, greeting the participant, asking questions on various subjects, sport, TV, etc. The purpose is to allow the collection of basic information that would be used in the second and third experiments for ERWIN to misattribute.

In the second experiment, the participants were categorized into two groups, one group with ERWIN remembering and making general conversation and the second group with ERWIN misattributing information. In both cases, the participants were given questionnaires to find out which group were happier with ERWIN.

In the third experiment, the participants from the previous experiment's non-misattributed set were given misattributed conversations and vice versa. At the end, all participants answered the same questionnaire to find out what type of characteristics in ERWIN participants liked the most.

\section{Data Collection \& Result Analysis:}

At the beginning of the first experiment the participants were given a sheet with five different pictures of ERWIN and had to identify the correct emotion expression from six corresponding options. This identification demonstrates the participant's ability to recognize various emotions. As the participants had not seen ERWIN before they had to identifying the emotions on the basis of their own knowledge. The pictures on the sheet showed the emotion expressions happy, sad, shocked, surprise and angry and can be seen in Figure 1. After evaluating the collected data for each emotion expression, $57 \%$ of the participants had selected the correct emotion option for the corresponding emotion picture. $21 \%$ of the participants had minor problems identifying the correct emotion expressions, confusing the emotion expressions: shocked and surprise, angry and sad.
ERWIN's interaction dialogues are based on various human conversational moments. These dialogues include greetings, asking the participant's name, their likes and dislikes and if possible picking up a topic from several choices. For example, ERWIN asked the participants if they liked football. During the conversation as many details about the participant as possible were gathered such as the color of their shirt, hair, gender, interests, etc.

The questions were aimed to find out the participant's preference about the biased and unbiased interactions. Participants were asked to complete a set of 11 questions which were aimed at determining the likeability of the interaction with ERWIN.

To compute the collected data from the both experiments and merging them into graph, we analyzed based on each question and each participant. The reliability scores from the 11 questionnaires for the biased and unbiased interactions are 0.94 and 0.756 (Cronbach's Alpha) which are very high (as shown below). In our case, the high Cronbach's Alpha actually supports to add all the ratings to get the score and compare between biased interaction and non-biased interaction. The histogram graphs for biased and unbiased responses are shown below. As we can see in Fig.4, the Mean for biased data is 87.93 which is approximately 40 points more from the unbiased Mean (47.67) which tells us that for each question, participant's responses were average of 40 point (in our ratings 4) higher for biased than unbiased. It is clear in graphs (Fig. 3) that the biased responses lied between 60 and 110, whereas the unbiased responses lied between 20 and 70 .

The histogram (Fig. 4) shows the average differences between the responses from participants in biased and unbiased conversation. The graph shows the number of participants preferred the biased interaction over the unbiased interaction. The mean calculated is $\mathbf{4 0 . 2 7}$ which tells that there is average of 40 point differences in ratings in prefer to biased interactions. In the questionnaires, the rating options were between 1 and 10 , so in this case, the average of 40 points actually suggests that in each questions participants rated average of 4 points higher in biased interactions. From the calculations and graphs, it can be concluded that participants liked the biased robot interactions over the unbiased robot interactions.

The correlation between each of the pairs of variables has calculated in Paired Sample Correlation test. Because there are same questionnaires and same participants, the same people are measured twice. The correlation between the two sets of scores is $\mathbf{0 . 4 7 2}$. It can be said that the pattern of change is consistent for the each participants for each questions. In general, overall participants actually enjoyed the biased interactions.

The 2-tailed sig ( $\mathrm{p}$ value) came out as $<0.05$ which indicate the significance our collected data over large population. From the above $t$-statistic, $\mathrm{t}=16.024$ and $\mathrm{p}<0.001$, i.e. a very small probability of this result occurring by chance, under null hypothesis of no difference. So the null hypothesis is rejected, since $\mathrm{p}<0.05$.

So, there is strong evidence $(\mathrm{t}=16.024, \mathrm{p}<0.0001)$ of preferring biased robot interactions over non-biased interactions. In this data set, participants preferred biased 
ERWIN interactions, on average, by approximately 40.27 points (in our case 4 point). In $95 \%$ confident interval, we can see that lower and upper limits are 35.12 and 45.4, which means larger population can prefer the biased interaction by Mean 40 and in a range between 35 and 45 points for each question. Therefore, from the above statistical analyse, we can conclude that, misattribution biases affected our interactions experiments, and overall, participant's significantly liked the interactions using misattribution bias in ERWIN's speech. This experiment result confirms our hypothesis and motivate to examine more biases in robot's interactive behaviours in our future experiments.

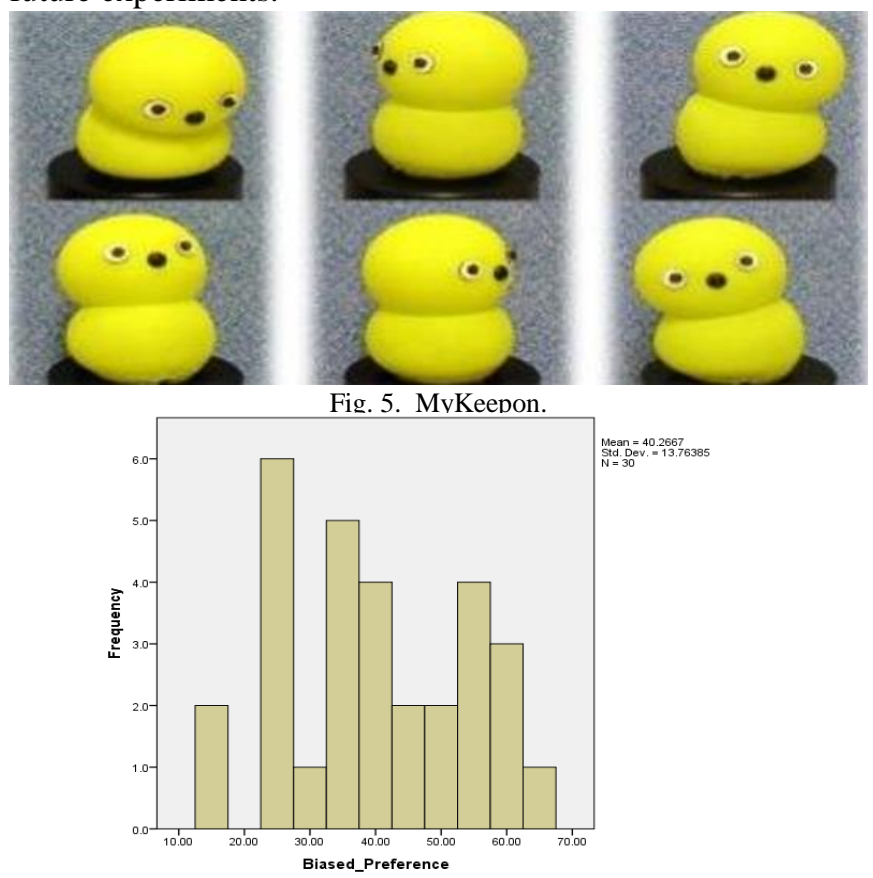

Fig. 3. Biased preference.

Paired Sample Test:

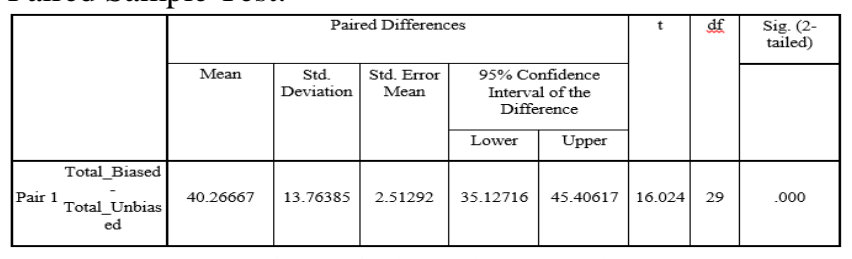

Fig 4. Paired Sample test Graph

\section{MyKeepon Experiments:}

Keepon is a small toy-like robot. It has 2 yellow coloured blobs on a black cylindrical base. The robot has 2 interactive modes, touch and dance. Keepon can make noises which represent various moods. User can touch its body or tap on its head, Keepon usually responds by circling around towards its touched side and makes noises and responds to taps on its head. The robot is very popular among children and has been used in many human-robot interaction experiments.

Open source software named 'ViKeepon' was used to remotely control the robot. With custom moves and sounds we created different interaction moments, such as, different greetings for two experiments, different dance movements, customized happy, sad and other expressions to make the robot more user friendly. Each participant was allowed upto
10 minutes of interaction with the MyKeepon. The interaction process included greetings, trying to establish eye contact with participant, responding to participant's actions, showing different movements, showing biased behaviours, showing sad expressions at the end of the interactions. Participants were allowed to touch and tap on the MyKeepon's head, also, they could clap to make Keepon dance. In general, number of times participant claps, the robot jumps same number of times. But, in biased interactions, we introduced empathy gap cognitive bias which allowed robot to become too happy if it jumped correct numbers of claps and too sad if it jumped wrong numbers of claps. In general, for the unbiased interactions the robot jumped correct numbers of the participant's claps and for the biased interactions it jumped wrong numbers of time and jumped fewer or more times.

MyKeepon became unresponsive during interactions to see the participants reactions in biased interactions. These type of different biased behaviours made the interaction different compared to unbiased interaction. In unbiased interaction, MyKeepon did not made mistakes in counting claps, or showing different behaviours. MyKeepon interacting with the participants without being unresponsive and the interaction followed very specific script, like, greeting, make the eye contact, showing different behaviours, jump when claps, be sad when participant leaves.

There were 2 experiments in this set. In the $1^{\text {st }}$ experiment, all the 30 participants interacted with unbiased MyKeepon and in the $2^{\text {nd }}$ experiment same 30 participants interacted with biased MyKeepon. The questionnaires were the same for both experiments to see the differences of the participant's likability to the interactions.

\section{E. Data Collection \& Result Analysis:}

The reliability scores from the 14 questionnaires for the biased and unbiased interactions are $\mathbf{0 . 8 7}$ and $\mathbf{0 . 8 3}$ (Cronbach's Alpha) which are high. In our case, the high Cronbach's Alpha actually supports to add all the ratings to get the score and compare between biased interaction and non-biased interaction. The histogram graphs (Fig.6) for biased and unbiased responses are shown below. The Mean for biased data is $\mathbf{5 5 . 3 6}$ which is approximately $\mathbf{3 . 0}$ point more from the unbiased Means (52.39) which tells us that for each question participant's responses were average of 2 ratings higher for biased than unbiased.

From the Paired Sample graph (Fig.7) we can see that the mean calculated is 2.97 which tells that there is average of 3 (approx.) point differences in ratings in prefer to biased interactions. The correlation between the two sets of scores is 0.95. It can be said that the pattern of change is consistent for the each participants for each questions.

From the above $t$-statistic, $t=8.032$ and $p<0.001$, i.e. a very small probability of this result occurring by chance, under null hypothesis of no difference. So the null hypothesis is rejected, since $\mathrm{p}<0.05$. According to the above measurements, there is strong evidence $(t=8.032, \mathrm{p}<0.0001)$ of preferring biased robot interactions over non-biased interactions. In this data set, participants preferred biased Keepon interactions, on 
average, by approximately 2.97 points. In $95 \%$ confident interval, we can see that lower and upper limits are 2.20706 and 3.72151, which means larger population can prefer the biased interaction by Mean of 2.97. Therefore, from the above statistical analyse, we can conclude that, used cognitive biases actually affected the interaction between the robot and participants, and overall, participant's liked the interactions using focusing effects and empathy gap biases in MyKeepon. This experiment result confirm our hypothesis and motivate to examine more biases in robot's interactive behaviours in our future experiments.

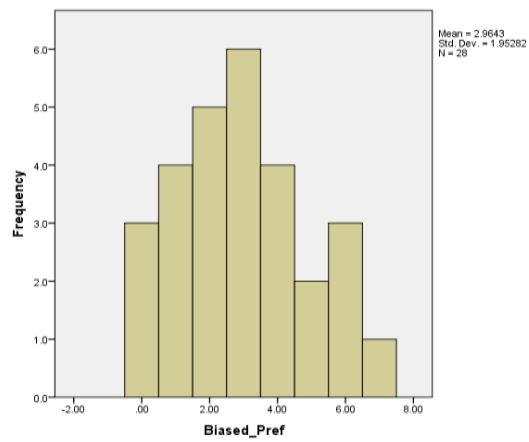

Fig. 6. Biased preference.

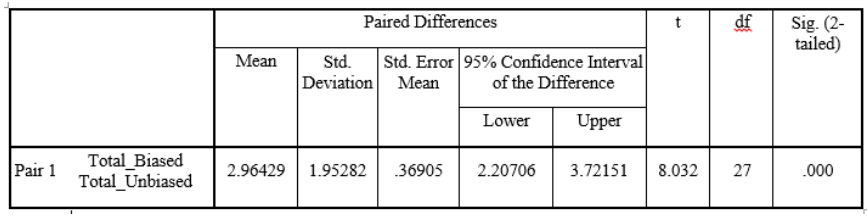

Fig 7. Paired Sample test Graph

\section{DISCUSSIONS}

The first experiments with ERWIN show that robots with general 'misattributes' bias is more likely to get human attention therefore become more effective in making relationships with humans. ERWIN's interaction dialogues were designed based on various human conversational moments and predefined topics. The $2^{\text {nd }}$ interactions, the conversations topics between biased and unbiased groups were almost the same, with the misattribution group having ERWIN intentionally repeat basic information incorrectly to the participants, for example, "Last time you were wearing a yellow shirt, am I correct?" and when the participant disagreed, ERWIN responded with "I am sorry that I have forgotten that, but I don't have true sense of colour perception. Next time I will be more careful though. " executing both sorry and surprised expressions simultaneously. For the remembered group, ERWIN simply repeated the same conversation but without misattributing the original information, for example, "Last time you were wearing a blue shirt"

In the biased interactions, the participants found it very surprising to see that the robot actually failed to remember their basic information including names and interests and also, the robot was confused and mixing up information while making conversation. Participant's reactions showed that they were very surprised and enjoyed the fact that a robot could indeed forget like humans. However, in the unbiased conversations, participants reacted normal as they were expecting that the robot would say their information correctly as they told to the robot in the previous interaction.

In the experiments using MyKeepon, participants responded similarly to the previous experiment. ERWIN uses verbal abilities with expressions were as MyKeepon uses movement and various noises to interact with the participants. The collected data from both experiments show participants initially liked both interactions but they preferred biased robots over unbiased. The biases used in MyKeepon were different, (empathic gap, conjunction, gambler fallacy, attentional bias etc.) so the robot had to show that it was expressing biased behaviors. As stated earlier, MyKeepon tried to make clear eye contact in the unbiased experiment, but not in the biased experiment.

Additionally MyKeepon jumped the wrong number of times to the responses of participant's pokes and became unresponsive, making sad noises, pointing its head towards the ground so as to express sadness as a result of making too many mistakes. At the beginning and ending of the biased interaction, MyKeepon showed overjoy and overly sad behaviors which were different from unbiased interaction.

According to participant responses, in the $1^{\text {st }}$ MyKeepon experiment (unbiased), they connected with the robot for its unique interactive behaviors and its toy like shape, but the $2^{\text {nd }}$ experiment (biased) the behaviors made the interactions more interesting to the participant. MyKeepon's expressions are already adorable (beatbote.net, 2014), but when it comes after making mistakes in counting or mistakes in predicting the directions, MyKeepon becomes sad, pointing its head to the ground, making sad noises - the whole situation gets very appealing to the participants.

Our two set of experiments shows that in both cases the unbiased interactions were favorable to the participants. These experiments actually answer one of our research questions, which was, "Despite a robot's appearances and its own functions and features, can human-like biased behaviours develop a human-like cognitively imperfect robot?" In our experiments, we can see that participants were able to develop preliminary attachments with the unbiased robots, but the robot's biased behaviors made the interactions more interesting to them. The participants felt more intimate with the robots when robots made mistakes and showed imperfect activities during interactions. As we mentioned earlier, human's perceptions towards robot affected by various science fictions and movies. In general, there is a conflict between the people's perceptions from literature, science fiction movies and the goal of the HRI researches (Sandoval E, 2014). Our experiments shows that, cognitive biases can be useful to reduce that conflict by making the robots cognitively imperfect. In our experiments participants experienced a different and completely new behaviors from our robots such as making mistakes, misattributing information, overly sad/ joy which could be unexpected to them.

It can be said that robots should have human-like faults, characteristic biases and prone to carry out common mistakes that humans make on a regular social basis - which will 
develop the robot's own characteristics and should lead to the acceptance of a robot for long-term interaction. It is expected that cognitive characteristics and personality in robots will make it easy for people to relate. Our experimental results show that participants enjoyed and developed a preferred relationship faster with a biased robot than the robot without bias. In the experiments with ERWIN it shows how one simple cognitive memory bias 'misattribution' was able to develop a better interaction with participants than the non-biased.

We understand that robot's biased interactive (such as, ERWIN's misattribution) factors actually relies on the robot itself, i.e., the way ERWIN communicates. Despite all the limitations, almost all the participants rated higher for the biased interactions than non-biased interactions in the both ERWIN and Keepon experiments, so it's safe to say that participants clearly preferred biased interactions over the unbiased interactions.

\section{CONCLUSION}

Our two case studies show that long-term relations could be possible between human and robot if the robot shows typical human-like imperfect behaviours in interactions. In human psychological nature, it is easy to interact with another humanlike personality that shows typical social characteristics (e.g. pet animals) (Meerbeek B, 2009). Robots in the other hand have abilities to perform human-like actions, can be designed to 'look' like humans and can appear to behave in a human like manner, but they lacks human-like cognitive personalities. Aristotle (384-322BCE), Immanuel Kant (1724-1804) have argued that human characters and personality can be described as imperfectly perfect (Stanford Encyclopedia, 2008), where robots lack to present such type of cognitive characteristics like unintentional mistakes, wrong assumptions, extreme presence of specific traits, task imperfectness and other human-like cognitive characteristics.

In social robotics, robots imitate human social queues like eye-gazing, human-like walking, talking and body moves etc. But the behavioural neutrality in humans is still missing in social robots in order to create and maintain long-term companionship beyond any time limit (Baxter P, 2012).

In our research, the cognitive biases in robot's behaviours suggest to express cognitive imperfectness, such as, judgemental mistakes, wrong assumptions, expressing tiredness, boredom or overexcitements, or scared of darkness and many other humanlike common characteristics. Sometime simple conventions may not be easy even in human interactions. Telling a joke well is a skill that few have, but also, telling a joke poorly is another human behaviour and may open more doors for further conversations. Perhaps the human-robot interactions experience is more troubling because someone that doesn't feel pain cannot be controlled or made to operate in the community normative. It is difficult to have a relationship with something that is too superior to us, and pretend to be too perfect without having any mistakes, faults which are unlike humans. The same is true even for humans. Even some people with different emotions are sometime alien to us. We expect, if a robot can show similar type of imperfections as humans in their behaviours, then the robots could be accepted to the majority of our society.

\section{REFERENCES}

1. Baron J, Thinking and Deciding, Fourth edition 2008, ISBN-13 978-0-521-86207-3

2. Bernt Meerbeek, Martin SaerbeckL, Christoph Bartneck, "Iterative design process for robots with personality", (2009). Iterative Design Process for Robots with Personality. Proceedings of the AISB2009 Symposium on New Frontiers in Human-Robot Interaction Edingburgh pp. 94-101.

3. Bless, H., Fiedler, K., \& Strack, F., "Social cognition: How individuals construct social reality", 2004, Hove and New York: Psychology Press

4. Biswas M, Murray J, "Effect of cognitive biases on human-robot interaction: A case study of a robot's misattribution", Robot and Human Interactive Communication, 2014 RO-MAN: The 23rd IEEE International Symposium, pp. 1024-1029.

5. Brian R. Duffy, "Anthropomorphism and The Social Robot", Robotics and Autonomous Systems 42, 2003, 177-190

6. Byron Reeves, Clifford Nass, "Perceptual Bandwidth, Communication of the ACM", March 2000/Vol. 43, No. 3

7. Cynthia Breazeal. "Social Interaction in HRI: The Robot View". IEEE, Transactions on Cybernetics, Man and Systems-Part C, Application and Review, May 2004. Vol 34 No. 2Cynthia L. Breazeal, Toward Sociable Robots, 2002

8. Cynthia L. Breazeal, "Designing Sociable Robots", The MIT Press, Cambridge, Massachusetts, ISBN 0-262-02510-8, 2001

9. Eduardo B. Sandoval, Omar Mubin, Mohammad Obaid, "Human Robot Interaction and Fiction: A Contradiction", Social Robotics Lecture Notes in Computer Science Volume 8755, 2014, pp 54-63.

10. Frank Tobe, 4 reports predict steady growth for robotics, http://www.therobotreport.com/news/four-research-reportspredict-steady-growth-for-robotic-segments, as of 18/12/ 2014

11. Hideki Kozima et al, "Keepon", International Journal of Social Robotics January 2009, Volume 1, Issue 1, pp 3-18

12. John C Murray et al, "MIRA: A Learning Multimodal Interactive Robot Agent", Hybrid Intelligent Systems, 2008. HIS '08. Eighth International Conference, DOI 10.1109/HIS.2008.137, 947-950

13. Kerstin Dautenhahn, "Methodology \& Themes of Human-Robot Interaction: A Growing Research Field", International Journal of Advanced Robotic Systems, Vol. 4, No. 1 (2007)

14. Kwan Min Lee, Wei Pen, Seung-A Jin, Chang Yan, "Can Robots Manifest Personality?: An Empirical Test of Personality Recognition", Social Responses, and Social Presence in Human-Robot Interaction, Journal of Communication, 2006, ISSN 0021-9916

15. Lilia Moshkina et al, "Time-varying affective response for humanoid robots, Progress in Robotics", Communications in Computer and Information Science Volume 44, 2009, pp 1-9

16. Maccoun, Robert J. (1998). "Biases in the interpretation and use of research results". Annual Review of Psychology 49 (1): 25987.

17. Michael Tomasello, "The Human Adaption for Culture, Annual Review of Anthropology", Volume 28 (1999).

18. Michael L. Walters, Dag S. Syrdal, Kerstin Dautenhahn, René te Boekhorst, Kheng Lee Koay, "Avoiding the Uncanny Valley Robot Appearance, Personality and Consistency of Behavior in an Attention-Seeking Home Scenario for a Robot", Autonomous Robots Journal, Volume 24 Issue 2, February 2008, Pages 159 178

19. Nelly Ben Hayoun, "Imperfect robots", http://nellyben.com/archive/the-test-rig-for-the-soyuz-chair/, as of $23 / 12 / 2014$

20. Paul Baxter et al, "Long-Term Human-Robot Interaction with Young Users", in Proceedings of the IEEE/ACM HRI-2011 workshop on Robots interacting with children, Lausanne, 2011.

21. Stanford Encyclopaedia of Philosophy, Kant's Moral Philosophy, First published Mon Feb 23, 2004; substantive revision Sun Apr 6, 2008, And, http://www.webpages.uidaho.edu/ngier/103/aristotle.htm 